\title{
Controllability of the Schrödinger Equation via Intersection of Eigenvalues
}

\author{
Ugo Boscain* \\ SISSA-ISAS, via Beirut 2-4, 34014 Trieste (Italy) \\ E-mail: boscainesissa.it

\section{Riccardo Adami} \\ Dipartimento di Matematica, Università “La Sapienza”, Piazzale Aldo Moro 2, 00185 Roma \\ (Italy) \\ E-mail: adami@mat.uniroma1.it
}

\begin{abstract}
We introduce two models of controlled infinite dimensional quantum system whose Hamiltonian operator has a purely discrete spectrum. For any couple of eigenstates we construct a path in the space of controls that approximately steers the system from one eigenstate to the other. To this purpose we use the adiabatic theory for quantum systems, and therefore the strategy requires large times.
\end{abstract}

keywords: Quantum Control, Controllability of PDEs, Adiabatic Theory, $\delta$-like Interactions

Control Systems: Theory, Numerics and Applications

30 March - 1 April 2005

Rome

${ }^{*}$ Speaker. 


\section{Introduction}

The issue of designing an efficient transfer of population between different atomic or molecular levels is crucial in atomic and molecular physics (see e.g. [5, 13, 20, 24]). In the experiments, excitation and ionization are often induced by means of a sequence of laser pulses. From the point of view of mathematics, the description of such processes translates into the problem of controlling the Schrödinger equation.

In recent years, such a problem has attracted increasing attention, in both communities of control theorist ([6, 23]), and experts in quantum dynamics (see for instance [9, 10]).

Many results are available in the case that the Hilbert space of the states of the system has finite dimension (see e.g. [7, 8, 11, 18] and references therein). Despite that, only few controllability properties have been proven for the Schrödinger equation as a PDE ([6, 23, 28]) and in particular no satisfactory global controllability results are available.

As a matter of fact, the controllability of the Schrödinger equation is recognized to have remarkable applications to Nuclear Magnetic Resonance as well as to Quantum Information, and to be a challenging mathematical problem.

In this paper we introduce two toy models and propose a method to prove approximate controllability of the Schrödinger equation. More specifically, given two arbitrary eigenstates of the uncontrolled system, we construct a path in the space of controls that steers the system from the first to the second; the target is reached only approximately, but the accuracy of the approximation can be arbitarily improved slowing the process down and correspondingly raising its duration. Our main technical tool is the adiabatic theorem ([4, 16, 21, 26]), which requires slowly varying controls and gives explicit estimates of the error.

It is worth pointing out that in order to apply our method we need a Hamiltonian with purely point spectrum that degenerates for some values of the controls. This seems to be in contradiction with the claimed use of the the adiabatic theory, which requires that during the whole time evolution the eigenvalues remain separated by a non vanishing gap ("gap condition").

The main idea is that such a difficulty can be overcome by a decoupling between the levels other than the adiabatic one. This observation is crucial in our analysis and we shall specialize it to the two toy models we deal with.

Before doing that, we stress that our strategy can be applied in many situations in which classical control theory would be too difficult or cumbersome. Besides, it provides explicit expressions of controls (motion planning), and most of all is very robust, in the sense that similar controls produce similar population transfers (see for instance [14, 27]). This last feature is very important in the experiments.

Let us describe our two models. The former is the simplest generalization to infinite dimension of three-level models that describe STIRAP processes (see for instance [14, 27]). As in that cases, it is given in the representation of the eigenfunction of the uncontrolled Hamiltonian, namely as an infinite dimensional matrix.

The full Hamiltonian reads

$$
H(u, v)=H_{0}+u B_{1}+v B_{2},
$$


where the drift (or "free") Hamiltonian $H_{0}$ has discrete spectrum and shows no degeneracies. The couplings $B_{1}$ and $B_{2}$ couple levels $E_{i}$ and $E_{i+1}$ for $i$ even and odd, respectively.

For every value of the real controls $u$ and $v$ the spectrum of $H(u, v)$ is still discrete, but degeneracies occur at isolated points in the space of the controls. This phenomenon holds generically for Hamiltonians depending on two parameters, and one refers to it as to the "conical crossing" of eigenvalues (see e.g. [15]).

Assume that at time zero $u=v=0$ and the system lies in the ground state of the drift $H_{0}$. The adiabatic theorem asserts that, employing slow varying controls $u(\varepsilon t)$ and $v(\varepsilon t)$ such that for any $t H(u(\varepsilon t), v(\varepsilon t))$ has no degeneracies, then at time $t$ the system lies close to the ground state of $H(u(\varepsilon t), v(\varepsilon t))$.

As widely known, the situation becomes more complicated when the system is driven near eigenvalues intersections.

Nevertheless, we exhibit paths in the control space that pass exactly through an eigenvalue intersection and force the system to perform a transition from the old to a new level. In Fig. 3 an example of a path steering the system from $E_{0}$ to $E_{2}$ is portrayed. Notice that, in order to employ such a strategy, we need to move controls along a surface, so we must have at our disposal at least two controls: the crucial point is to cross the eigenvalues intersections in one direction only, and this would not be possible with one control.

The strategy presented here is inspired to a numerical method developed for finite dimensional systems in [27].

Although this toy model is easily solvable using classical control theory in our opinion it clearly illustrates how our method works.

The second model consists of the Schrödinger picture of a quantum particle in a one-dimensional infinite potential well with some additional controlled external fields.

The main obstacle to be overcome is that in a one dimensional quantum system the presence of degeneracies in the discrete spectrum is a highly nonstandard feature. In particular the non degeneracy of the ground state holds in any dimension for systems subject to a locally integrable potential field ([19]). Therefore our strategy consists in producing degeneracies by means of potentials with non integrable singularities. To this purpose we use point interaction potentials (Dirac $\delta$ and $\delta^{\prime}$ ) with a strength to be sent to infinity. Let us recall that interactions like Dirac $\delta$ and $\delta^{\prime}$ are widely used in modeling of quantum system, since Fermi's paper [12] up to contemporary applications [1, 2, 3].

We consider a particle confined to the interval $(-\pi / 2, \pi / 2)$, whose Hamiltonian reads

$$
\begin{aligned}
H(u, v, w):= & -\partial_{x}^{2}+u \delta(x-\pi / 2)+v \delta^{\prime}(x-\pi / 2) \\
& +w \theta(x-\pi / 2)
\end{aligned}
$$

and the drift coincides with $H(0,0,0)=-\partial_{x}^{2}$.

Assume that at time zero the system lies in the ground state of the drift Hamiltonian and we switch a Dirac's delta interaction on, located at the center of the well, with strength $u(\varepsilon t)$. Then the energy of the ground state is slowly increasing with time, while the energy of the first excited level remains unchanged. In the limit $u(\varepsilon t) \rightarrow \infty$ the two energy levels coincide, but the associate eigenfunctions do not. We then use a Heaviside function in order to perform a rotation in the 
two dimensional eigenspace of the degenerate eigenvalue, and reach the eigenfunction of the first excited level of the drift Hamiltonian. In this way we obtain a transition from the ground level to the first excited.

In contrast with the previous model, here we exploit an intersection obtained letting the control $u$ diverge; however the Hamiltonian (1.1) is well defined also for an infinite value of $u$ and $v$.

Moreover, the gap condition is fulfilled because of the parity selection rule: the $\delta$ potential and the ground state of the drift are even, therefore even and odd levels are decoupled during the entire evolution and the effective gap is the one between the ground state and the second excited level.

Replacing the delta potential with a "delta prime" interaction, one can repeat this procedure and induce a transition from the first excited state to the second; more generally, alternating delta and delta prime one can reach any energy level. This control strategy can be generalized to any symmetric (coercive) potential.

It is worth mentioning that, unlike the first toy model, in this case it seems extremely difficult to prove that it is possible to steer the system from two eigenstates using classical control theory (for instance using finite dimensional techniques on a Galerkin approximation of the system, and then passing to the limit).

\subsection{Definitions of Controllability}

Let us introduce the notions of controllability that we need in the following.

Definition 1.1. Consider a quantum mechanical system whose evolution is described by a self adjoint Hamiltonian depending on $m$ real parameters in the form:

$$
H\left(u_{1}, \ldots u_{m}\right)=H_{0}+u_{1} B_{1}+\ldots+u_{m} B_{m} .
$$

Assume that for every value of the parameters $u_{1}, \ldots, u_{m}$ the spectrum of $H\left(u_{1}, \ldots u_{m}\right)$ is discrete. Assume moreover that the drift Hamiltonian $H(0, \ldots, 0)=H_{0}$ has non degenerate spectrum $E_{0},<$ $E_{1}<E_{2}<\ldots$ with $\Phi_{0}, \Phi_{1}, \ldots$ the corresponding eigenvectors.

We say that such a system is:

- finite-time state to state controllable ( $f$-SSC for short) in the class $\mathcal{K}$ if for every $j, l \in \mathbb{N}$ there exist open loop controls $u_{1}(),. \ldots, u_{m}(.) \in \mathcal{K}$ steering the system from $\Phi_{j}$ to $\Phi_{l}$ in finite time $T(j, l)$.

- approximately state to state controllable (a-SSC for short) in the class $\mathcal{K}$, if for every $j, l \in \mathbb{N}$ and $\varepsilon>0$ there exists a time $T(j, l, \varepsilon)$ and open loop controls $u_{1}(),. \ldots, u_{m}(.) \in \mathcal{K}$, steering the system from $\Phi_{j}$ to a state $\Phi_{a p p}$ arbitrarily close to the space spanned by $\Phi_{l}$. Namely, there exists $\varphi \in[0,2 \pi[$ such that

$$
\left\|e^{-i \varphi} \Phi_{a p p}-\Phi_{l}\right\|_{L^{2}} \leq \varepsilon
$$

It is widely recognized that, in general, using a finite number of controls on an infinite dimensional system, one can expect only approximate controllability, and in our case we prove that our toy models are a-SSC. As already mentioned, thanks to its special form the first model is f-SSC, but this seems not true for more general systems, and in particular for the second one. 


\subsection{The Adiabatic Theorem}

Here we recall some basic ideas on the adiabatic theorem; an exhaustive introduction to this topic together with a general formulation of the theorem can be found in the first and second chapters of [26].

It is quite obvious that in quantum systems governed by a time-independent Hamiltonian the evolution preserves the eigenvectors of the energy. On the other hand, if the Hamiltonian depends on time this is in general not true. The adiabatic theorem states that, provided that the time-dependence of the Hamiltonian is suitably slow, then the eigenvectors are approximately preserved.

More precisely, let $H(\varepsilon t)$ be a slowly time-dependent Hamiltonian with purely discrete spectrum, $\lambda_{j}(\varepsilon t)$ its $j^{\text {th }}$ eigenvalue and $P_{j}(\varepsilon t)$ the orthogonal projection on the space $\mathcal{H}_{j}(\varepsilon t)$ of the eigenvectors associated to $\lambda_{j}(\varepsilon t)$.

Clearly, the time evolution $U_{\varepsilon}(t, s)$ generated by $H(\varepsilon t)$ preserves $\mathcal{H}_{j}(\varepsilon t)$ if and only if it fulfils the following intertwining property:

$$
P_{j}(\varepsilon t) U_{\varepsilon}(t, s)=U_{\varepsilon}(t, s) P_{j}(\varepsilon s) .
$$

Since

$$
\frac{d}{d t}\left(U_{\varepsilon}(s, t) P_{j}(\varepsilon t) U_{\varepsilon}(t, s)\right)=\varepsilon U_{\varepsilon}(s, t) \dot{P}_{j}(\varepsilon t) U_{\varepsilon}(t, s)
$$

where the dot denotes the derivative w.r.t. $t$, then relation (1.3) is satisfied at zero order in $\varepsilon$ only. In fact, (1.3) is exactly satisfied by the evolution $U_{a}^{\varepsilon}(t, s)$ generated by the so-called "adiabatic Hamiltonian" associated to the $j^{\text {th }}$ level, that reads

$$
H_{a}(\varepsilon t):=H(\varepsilon t)-2 i \varepsilon P_{j}(\varepsilon t) \dot{P}_{j}(\varepsilon t)+i \varepsilon \dot{P}_{j}(\varepsilon t)
$$

The adiabatic theorem estimates the difference between the time evolutions $U_{\varepsilon}$ and $U_{a}^{\varepsilon}$.

Theorem 1.2. Consider a family $H(t)$ of self adjoint operators on a Hilbert space $\mathcal{H}$, with $t$ in the possibly unbounded interval $\left(t_{1}, t_{2}\right)$. Suppose that:

1. all $H(t)$ 's have a common dense domain $\mathcal{D}$.

2. $H(\cdot) \in \mathcal{C}_{b}^{2}\left(\left(t_{1}, t_{2}\right), \mathcal{L}(\mathcal{D}, \mathcal{H})\right)$, where $\mathcal{L}(\mathcal{D}, \mathcal{H})$ denotes the space of bounded linear operators from $\mathcal{D}$ to $\mathcal{H}$ provided that $\mathcal{D}$ is endowed with the norm of the graph of $H\left(t_{1}\right)$ :

$$
\|T\|_{\mathcal{L}(\mathcal{D}, \mathcal{H})}:=\sup _{v \in \mathcal{D} \backslash\{0\}} \frac{\|T v\|_{\mathcal{H}}}{\|v\|_{\mathcal{H}}+\left\|H\left(t_{1}\right) v\right\|_{\mathcal{H}}}
$$

3. for every $t$, the spectrum $\sigma(H(t))$ of $H(t)$ is discrete and non degenerate, i.e. $\sigma(H(t))=$ $\left\{\lambda_{j}(t), j=0, \ldots, n, \ldots, \lambda_{i}(t)<\lambda_{k}(t)\right.$ if $\left.i<k\right\}$.

4. Fixed $j \in \mathbb{N}$, the following gap condition is satisfied: 


$$
g:=\inf _{t \in\left(t_{1}, t_{2}\right)} \min \left(\lambda_{j+1}(t)-\lambda_{j}(t), \lambda_{j}(t)-\lambda_{j-1}(t)\right)>0
$$

Let $U_{\varepsilon}$ and $U_{a}^{\varepsilon}$ be the two-parameter propagators generated by $H(\varepsilon t)$ and $H_{a}(\varepsilon t)$ defined in (1.4) respectively. Then, for any $t$ and $t_{0}$ in $\left(t_{1}, t_{2}\right)$,

$$
\left\|U_{\varepsilon}\left(t, t_{0}\right)-U_{a}^{\varepsilon}\left(t, t_{0}\right)\right\|<C \varepsilon\left(1+\varepsilon\left|t-t_{0}\right|\right)
$$

where the constant $C$ diverges for vanishing $g$.

Notice that if at time $t_{0}$ the system lies in an eigenstate of $H\left(\varepsilon t_{0}\right)$ associated to the eigenvalue $\lambda_{j}\left(t_{0}\right)$, then estimate (1.5) gives

$$
\left\|\psi_{\varepsilon}(t)-\psi_{a}^{\varepsilon}(t)\right\|<C \varepsilon\left(1+\varepsilon\left|t-t_{0}\right|\right)
$$

where $\psi_{\varepsilon}(t)$ represents the actual state of the system and $\psi_{a}^{\varepsilon}(t)$ is eigenvector of $H(\varepsilon t)$ relative to the eigenvalue $\lambda_{j}(\varepsilon t)$.

Furthermore, if the $j^{\text {th }}$ level of $H(t)$ is non degenerate at any time $t$, and $\Phi_{j}(t)$ is the associated eigenvector, then

$$
H_{a}(\varepsilon t) \Phi_{j}(\varepsilon t)=\lambda_{j}(\varepsilon t) \Phi_{j}(\varepsilon t)-i \varepsilon \dot{P}_{j}(\varepsilon t) \Phi_{j}(\varepsilon t)
$$

and

$$
U_{a}^{\varepsilon}\left(t, t_{0}\right) \Phi_{j}\left(\varepsilon t_{0}\right)=\exp \left(-i \int_{0}^{t} d s \lambda_{j}(\varepsilon s)\right) \Phi_{j}(\varepsilon t)
$$

The paper is organized as follows. In Section 2 we present the first toy model. After studying the spectrum of the Hamiltonian, we introduce the concept of climbing path and prove that the adiabatic theorem can be applied to climbing paths even if the gap condition is not satisfied (see Theorem 2.6). As a corollary we get that the system is a-SSC (see Corollary 2.7). In Section 3 , we present the second model. In this case, using locally non integrable controlled potentials, we prove that the system is a-SSC (see Theorem 3.2).

\section{An Infi nite Dimensional Toy Model}

In this section, we introduce an infinite dimensional model which generalizes in a natural way the features of a finite dimensional quantum mechanical system in interaction with two external fields. After introducing the model, we describe the spectrum of the Hamiltonian operator and show how to apply the adiabatic theory to prove a-SSC.

\section{The model and the spectrum of the Hamiltonian.}

Let us consider an infinite dimensional quantum system endowed with a purely discrete spectrum and suppose that its energy levels are non degenerate. Let $E_{j}$ be the energy of the $j$.th level with $E_{j}<E_{k}$ for $j<k$ and let $E_{0}$ be the energy of the ground state. In the basis of the eigenstates of the energy, the Hamiltonian is represented by an infinite diagonal matrix, whose $j^{\text {th }}$ element equals $E_{j}$. In the following we refer to this Hamiltonian as to the drift or free Hamiltonian. 
We have at our disposal two real controls $u$ and $v$ that couple energy levels by pairs in such a way that the infinite matrix representing the controlled Hamiltonian reads

$$
H(u, v)=\left(\begin{array}{cccccc}
E_{0} & \alpha_{0} u & 0 & 0 & 0 & \cdots \\
\alpha_{0} u & E_{1} & \beta_{0} v & 0 & 0 & \cdots \\
0 & \beta_{0} v & E_{2} & \alpha_{1} u & 0 & \cdots \\
0 & 0 & \alpha_{1} u & E_{3} & \beta_{1} v & \cdots \\
\vdots & \vdots & \vdots & \vdots & \vdots & \vdots \\
\vdots & \vdots & \vdots & \vdots & \vdots & \vdots
\end{array}\right)
$$

Here the coefficients $\alpha_{j}$ 's and $\beta_{j}$ 's (that we assume to be bigger than zero) implement the fact that different pairs of levels react in different manners to the presence of the external fields. Therefore, such coefficients are to be considered characteristic of the system and represent the strength of the coupling induced by the external fields on the concerned pairs of levels.

The matrix (2.1) defines a linear operator $\hat{H}(u, v)$ acting on the Hilbert space $\ell^{2}$ of all complex sequences $\left\{x_{j}\right\}$ such that $\sum_{j=0}^{\infty}\left|x_{j}\right|^{2}<\infty$.

Moreover, for technical reasons we assume that the sequence of the $E_{j}$ 's diverges, and the quantities $\alpha_{j} /\left|E_{2 j}\right|^{\mu}$ and $\beta_{j} /\left|E_{2 j}\right|^{\mu}$ vanish as $j$ goes to infinity for some $0<\mu<1$.

These hypotheses yield some remarkable consequences in terms of spectral properties of $\hat{H}(u, v)$, namely:

Proposition 2.1. Under the hypotheses previously stated on the coefficients $E_{j}, \alpha_{j}, \beta_{j}$, the operator $H(u, v)$ defined in (2.1) and acting on $\ell^{2}$, satisfies the following:

(i) $\hat{H}(u, v)$ is self-adjoint.

(ii) The spectrum of $\hat{H}(u, v)$ is purely discrete.

(iii) If both $u$ and $v$ are different from zero, then all eigenvalues are non degenerate.

(iv) The spectrum of $\hat{H}(u, v)$ is equal to the spectrum of $\hat{H}(|u|,|v|)$.

We omit proofs, but just remark that statements (i) and (ii) follow from general topics in spectral analysis ([22]) while (iii) and (iv) can be proven by direct inspection. Furthermore, due to (iv) we shall limit ourselves to non negative controls $u$ and $v$.

Owing to (i), (ii) and (iii) and using perturbation theory [17], one can prove that there exists a unique countable set of continuous functions $\lambda_{j}: \mathbb{R}^{2} \rightarrow \mathbb{R}$ such that

A. $\lambda_{j}(u, v)$ is an eigenvalue of $\hat{H}(u, v)$.

B. $\lambda_{j}(u, v) \leq \lambda_{j+1}(u, v)$ for any $j \in \mathbb{N}, u, v \in \mathbb{R}$ and $\lambda_{j}(u, v)<\lambda_{j+1}(u, v)$ for any $j \in \mathbb{N}, u, v>0$.

Roughly speaking, the graph of the spectrum of $\hat{H}(u, v)$ as a multi-valued function of $u$ and $v$ can be represented as a countable family of regular surfaces possibly intersecting along the axes $u=0$ and $v=0$ only. 
In order to steer the system from two fixed eigenstates using adiabatic theory, it is essential to classify all possible eigenvalues intersections.

Intersections along the axis $u=0$.

The matrix $H(0, v)$ is block diagonal. The first block is $1 \times 1$ and consists of the element $E_{0}$, while the others are $2 \times 2$ and take the form:

$$
\mathbf{B}_{j}:=\left(\begin{array}{cc}
E_{2 j+1} & \beta_{j} v \\
\beta_{j} v & E_{2 j+2}
\end{array}\right), \quad j=0,1,2, \ldots
$$

The eigenvalues of $\mathbf{B}_{j}$ are given by

$$
\left\{\begin{array}{l}
\Lambda_{j}^{+}(v)=E_{2 j+2}+\Delta_{j} \\
\Lambda_{j}^{-}(v)=E_{2 j+1}-\Delta_{j}
\end{array}\right.
$$

where the quantity

$$
\Delta_{j}:=\frac{\sqrt{\omega_{j}^{2}+4 \beta_{j}^{2} v^{2}}}{2}-\omega_{j}
$$

with

$$
\omega_{j}:=E_{2 j+2}-E_{2 j+1}
$$

representing the exchange of energy induced by the coupling between the two levels. Notice that turning the control $v$ on increases the energy of the even levels while lowers that of the odd ones. Then, the energy gap between two coupled levels is enhanced, as shown in Fig. 1.

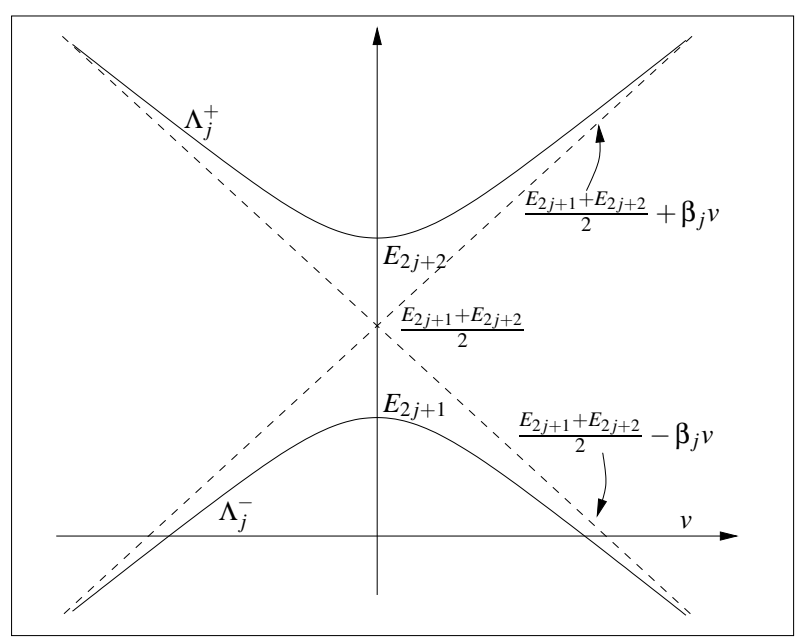

Figure 1

Equalities (2.3) provide some simple rules in order to classify all crossing of eigenvalues.

Set $\Lambda_{-1}^{+}:=E_{0}$ and $\beta_{-1}:=0$. Consider a pair of indices $j$ and $k$, with $j>k$. Then

- There exists a unique $v>0$ such that $\Lambda_{j}^{+}(v)=\Lambda_{k}^{-}(v)$. Conversely, the equation $\Lambda_{j}^{-}(v)=$ $\Lambda_{k}^{+}(v)$ has no solutions. 
- If $\beta_{j}<\beta_{k}$, then there exists a unique $v>0$ such that $\Lambda_{j}^{+}(v)=\Lambda_{k}^{+}(v)$. Conversely, if $\beta_{j} \geq \beta_{k}$ then the graphs of the functions $\Lambda_{j}^{+}$and $\Lambda_{k}^{+}$do not intersect each other.

- If $\beta_{j}>\beta_{k}$, then there exists a unique $v>0$ such that $\Lambda_{j}^{-}(v)=\Lambda_{k}^{-}(v)$. Conversely, if $\beta_{j} \leq \beta_{k}$ then the graphs of the functions $\Lambda_{j}^{-}$and $\Lambda_{k}^{-}$do not intersect.

Intersections along the axis $v=0$.

The matrix $H(0, v)$ is block diagonal, each block being $2 \times 2$ and taking the form:

$$
\mathbf{A}_{j}:=\left(\begin{array}{cc}
E_{2 j} & \alpha_{j} u \\
\alpha_{j} u & E_{2 j+1}
\end{array}\right), \quad j=0,1,2, \ldots
$$

The eigenvalues of $\mathbf{A}_{j}$ are given by

$$
\Gamma_{j}^{-}(u)=E_{2 j}-D_{j} \Gamma_{j}^{+}(u)=E_{2 j+1}+D_{j}
$$

with

$$
\begin{aligned}
& \mathcal{D}_{j}:=\frac{\sqrt{\Omega_{j}^{2}+4 \alpha_{j}^{2} v^{2}}}{2}-\Omega_{j} \\
& \Omega_{j}:=E_{2 j+1}-E_{2 j}
\end{aligned}
$$

Like in the previous case, a complete classification of the eigenvalue intersections can be done.

Remark 2.2. We want to prevent the reader from identifying functions $\Lambda_{j}$ 's and $\Gamma_{j}$ 's with the $\lambda_{k}$ 's, even for suitable values of $k$ (for instance, $\Lambda_{j}^{+}(u)$ with $\lambda_{2 j+2}$ ). Such identification is correct if the controls are smaller than the lowest value for which a degeneracy occurs. This is due to the fact that the $\lambda_{i}$ 's satisfy $\lambda_{i}(u, v) \leq \lambda_{j}(u, v)$, for $i<j$ and for every $(u, v) \in \mathbf{R}^{2}$. While the graphs of the functions $\Lambda_{j}$ 's (resp. the $\Gamma_{j}$ 's) can cross each other. To clarify such important point we put in evidence in Fig. 2 the graph of the functions $\lambda_{0}(0, v), \lambda_{1}(0, v), \lambda_{2}(0, v), \lambda_{3}(0, v)$ and the corresponding identification with the $\Lambda_{i}$ 's, corresponding to a peculiar choice of the coefficients $\beta_{j}$ 's.

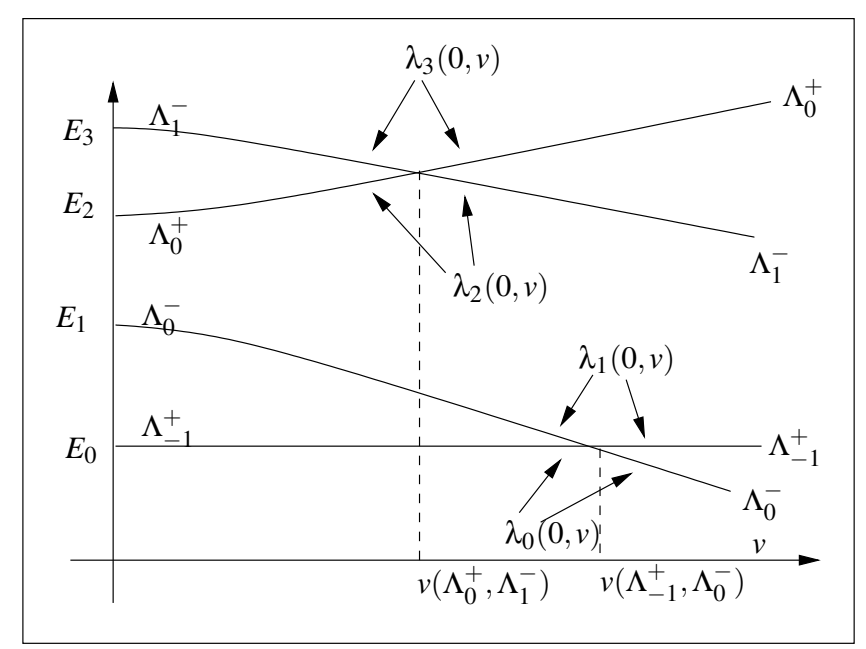

Figure 2 
The investigation undertaken in this Section can be resumed as follows.

Theorem 2.3. The set

$$
\mathcal{S}:=\{(u, v, p) \text { s.t. } u, v \in \mathbf{R}, p \text { eigenvalue of } \hat{H}(u, v)\}
$$

is the union of the graphs of a countable, increasing family of continuous functions $\lambda_{j}(u, v)$, i.e.

$$
\begin{aligned}
\mathcal{S} & =\cup_{j=0}^{\infty} \mathcal{S}_{j} \\
\mathcal{S}_{j} & =\left\{\left(u, v, \lambda_{j}(u, v)\right) \text { s.t. } u, v \in \mathbf{R}\right\}
\end{aligned}
$$

Such graphs intersect one another in a countable set lying in the union of the planes $u=0$ and $v=0$. Apart from that points, they are smooth.

Fig. 3 gives an idea of the shape of the three first surfaces belonging to $\mathcal{S}$, for some value of the parameters $E_{i}, \alpha_{i}$, and $\beta_{i}$, which could be useful to figure out how to employ the adiabatic theory (see below).

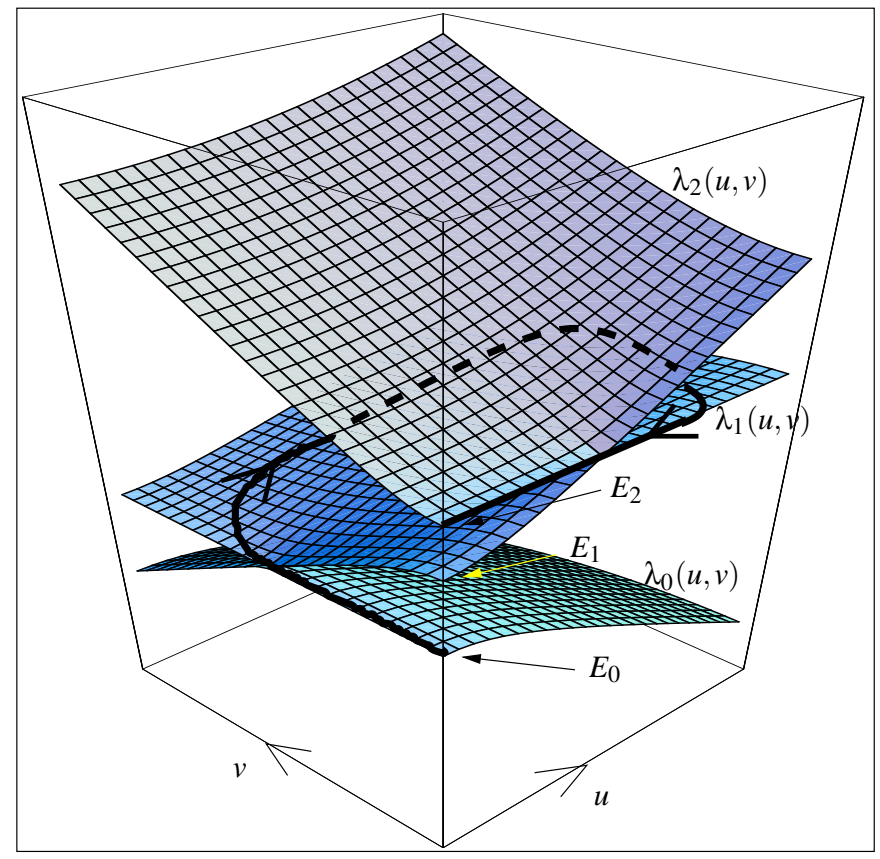

Figure 3

\section{Controllability via Classical Control Theory}

Let us consider the Schrödinger equation for the Hamiltonian (2.1):

$$
i \frac{d \psi(t)}{d t}=H(u(t), v(t)) \psi(t)
$$

where $\psi(t)=\left(\psi_{0}(t), \psi_{1}(t), \ldots.\right)$ is a vector in $\ell^{2}$. The crucial feature of this model is that if $u=0$ or $v=0$, then the variables $\psi_{1}, \psi_{2}, \ldots$ are coupled by pairs. More precisely we have the following: 
- If $u=0$ then $\psi_{0}$ is decoupled and $\psi_{i}$ is coupled only to $\psi_{i+1}$ for $i$ odd.

- If $v=0$ then $\psi_{i}$ is coupled only to $\psi_{i+1}$ for $i$ even.

As a consequence, using standard techniques of control theory on $S U(2)$, one can prove the following:

Proposition 2.4. If the functions $u(\cdot)$ and $v(\cdot)$ are measurable and essentially bounded functions then the system (2.1), (2.8) is $\mathrm{f}-\mathrm{SSC}$.

\section{Controllability via Adiabatic Theory}

In the following we prove a-SSF for this toy model, using adiabatic theory.

Consider a $C^{2}$ path $(u(\varepsilon t), v(\varepsilon t))$ in $\mathbf{R}^{2}$. Outside the set $u v=0$ the time evolution can be approximated by means of the adiabatic theorem. More precisely one can directly check that:

$$
\frac{d^{2}}{d t^{2}} H(u(\varepsilon t), v(\varepsilon t))=\varepsilon^{2}\left(\frac{d^{2} u}{d t^{2}}(\varepsilon t) B_{1}+\frac{d^{2} v}{d t^{2}}(\varepsilon t) B_{2}\right) .
$$

Now since $B_{1}$ and $B_{2}$ are relatively bounded with respect to $H_{0}$, then $H(u(t), v(t))$ belongs to $\mathcal{C}_{b}^{2}\left(\left(t_{1}, t_{2}\right), \mathcal{L}(\mathcal{D}, \mathcal{H})\right)$ and one can apply Theorem 1.2 .

Since the constant $C$ supplied by the adiabatic theorem (cfr. formula (1.5)) diverges as the gap vanishes, then the approximation we get in (1.2) is as good as the path $(u(\varepsilon t), v(\varepsilon t))$ stays far from the singularities.

In the following we describe what happens if the path crosses a singularity along one axis $u=0$ or $v=0$.

For the sake of clearness, let us suppose that Fig.2 represents the lowest three energy levels of $H(0, v)$ as functions of $v$.

Assume moreover that $(u(\varepsilon t), v(\varepsilon t))=(0, \varepsilon t)$ for $t \in\left[0, \varepsilon^{-1} T\right]$, and that, at time zero, the system lies in an eigenstate of $E_{2}$. We denote $\varepsilon^{-1} t_{0}$ the time at which the path crosses the singularity.

Since $H(0, v)$ is block diagonal, after time zero the eigenvalue $\Lambda_{0}^{+}$is coupled with $\Lambda_{0}^{-}$only. The adiabatic approximation can then be used and the gap reads $g=\inf _{v \in[0, T]}\left(\Lambda_{0}^{+}(v)-\Lambda_{0}^{-}(v)\right)=$ $E_{2}-E_{1}$.

Notice that, due to the decoupling between the levels $\Lambda_{0}^{+}$and $\Lambda_{1}^{-}$, the system follows $\Lambda_{0}^{+}$even after the path crosses the intersection with $\Lambda_{1}^{-}$. This is the crucial point in our procedure. Now, noticing that

$$
\Lambda_{0}^{+}(v)= \begin{cases}\lambda_{2}(0, v), & v \in\left[0, t_{0}[\right. \\ \lambda_{3}(0, v), & v \in] t_{0}, T[\end{cases}
$$

it follows that, at time $\varepsilon^{-1} t_{0}$, the system jumps on the level $\lambda_{3}$.

In the following we formulate in more detail this procedure. Consider the set $\mathcal{S}$ defined in (2.7). A point $(u, v, p) \in \mathcal{S}$ is called a singularity if $p=\lambda_{i}(u, v)=\lambda_{j}(u, v)$ with $i \neq j$. From the analysis preceding theorem (2.3), it follows that the singularities are isolated points and if $(u, v, p)$ is a singularity then $u v=0$. We denote by $Z$ the set of all singularities. 
Definition 2.5. Consider a map $\gamma(\cdot):=(u(\cdot), v(\cdot), p(\cdot)):[0, \tau] \rightarrow \mathcal{S} \subset \mathbf{R}^{3}$. We say that this map is a climbing path if:

- it is a $C^{2}$ map from $[0, \tau]$ to $\mathbf{R}^{3}$;

- $\gamma(0)=(u(0), v(0), p(0))=\left(0,0, E_{A}\right)$ and $\gamma(\tau)=(u(\tau), v(\tau), p(\tau))=\left(0,0, E_{B}\right)$ for some $A, B \in$ $\mathbf{N}$;

- it passes through a finite number of singularities. i.e. $\operatorname{Supp}(\gamma) \cap Z$ is finite.

- if $\tau_{1}, \ldots, \tau_{n}$ are the values of the parameter at which the singularities are met, namely $\gamma\left(\tau_{i}\right) \in Z$ for any $i$, then there exist intervals $\left[a_{i}, b_{i}\right]$ such that $\left.\tau_{i} \in\right] a_{i}, b_{i}[$ and $u$ or $v$ constantly vanishes on $\left[a_{i}, b_{i}\right]$.

An example of climbing path is represented in Fig. 3. If a climbing path is slowly gone along, then we can apply the adiabatic theorem and obtain the following result:

Theorem 2.6. Consider the family of Hamiltonians $H(u, v)$ and a climbing path $\gamma$ on the set $\mathcal{S}$ defined in (2.7). Given $\varepsilon \ll 1$ consider the following parametrization of $\gamma: \gamma(\varepsilon t)=(u(\varepsilon t), v(\varepsilon t), p(\varepsilon t))$, with $t \in[0, T]$ and $T:=\varepsilon^{-1} \tau$. Let $\Phi_{j}(u, v)$ be the eigenvector corresponding to the eigenvalue $\lambda_{j}(u, v)$. Let $t_{1}, \ldots, t_{n}$ be the times at which the singularities are met, namely $\gamma\left(\varepsilon t_{i}\right) \in Z$ for any $i$. Let $j_{i}$ be defined by $\left.\left.p(\varepsilon t)=\lambda_{j_{i}}(u(\varepsilon t), v(\varepsilon t)), t \in\right] t_{i}, t_{i+1}\right]$. Then, for every $\left.\left.t \in\right] t_{i}, t_{i+1}\right]$, we have

$$
\begin{aligned}
& \| \exp \left(i \int_{0}^{\varepsilon t} d s \lambda_{j_{i}}(u(s), v(s))\right) \Phi_{j_{i}}(u(\varepsilon t), v(\varepsilon t))-\psi(\varepsilon t) \| \\
&<C \varepsilon(1+\varepsilon|t|) \leq C \varepsilon(1+\tau)
\end{aligned}
$$

where $\psi(t)$ is the solution of the Schrödinger equation

$$
i \partial_{t} \psi(t)=H(u(\varepsilon t), v(\varepsilon t)) \psi(t)
$$

with initial data

$$
\psi(0)=\Phi_{j_{i}}(0,0)
$$

Roughly speaking this theorem states that if the singularities are crossed keeping to zero one of the two controls, then the adiabatic theorem holds true and the system jumps at the singularities from a level to another one. Notice that estimate given in theorem 2.6 holds for $t=T=\varepsilon^{-1} \tau$ also, with the system passed to the level of energy $E_{B}$. Therefore, we have the following

Corollary 2.7. The quantum mechanical system described by the Hamiltonian (2.1) is a-SSC in the class $C_{b}^{2}$.

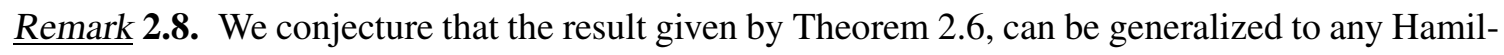
tonian having conical intersections. (see for instance [15]). 


\section{Controllability via Singular Potentials}

In this section we introduce an infinite dimensional model in the Schrödinger picture, mimicking the feature of the model analyzed in Sec. II.

We start considering an infinite one dimensional potential well with Dirichlet boundary conditions and add suitable controlled potentials in order to induce transitions between different energy levels.

As explained in the introduction our strategy consists in producing degeneracies using potentials with non integrable singularities. To this purpose we use point interaction potentials with a coupling constant to be sent to infinity.

Let us introduce the model. Consider the evolution problem on the space $L^{2}(0, \pi)$ given by

$$
\begin{aligned}
i \partial_{t} \psi(x, t)= & H(u(\varepsilon t), v(\varepsilon t), w(\varepsilon t)) \psi(x, t), \text { where: } \\
H(u, v, w):= & -\partial_{x}^{2}+u \delta(x-\pi / 2)+v \delta^{\prime}(x-\pi / 2) \\
& +w \theta(x-\pi / 2) .
\end{aligned}
$$

Here $\partial_{x}^{2}$ is the second partial derivative w.r.t. $x$ with Dirichlet boundary conditions (i.e. $\psi(0, t)=$ $\psi(\pi, t)=0)$. The term $u(\varepsilon t) \delta\left(x-\frac{\pi}{2}\right)$ is a Dirac delta potential whose effect results in the boundary condition

$$
\lim _{x \rightarrow \frac{\pi}{2}^{+}} \partial_{x} \psi(x, t)-\lim _{x \rightarrow \frac{\pi}{2}^{-}} \partial_{x} \psi(x, t)=u(\varepsilon t) \psi(\pi / 2, t) .
$$

Analogously, the term $v(\varepsilon t) \boldsymbol{\delta}^{\prime}\left(x-\frac{\pi}{2}\right)$ corresponds to the boundary conditions

$$
\begin{gathered}
A(t):=\lim _{x \rightarrow \frac{\pi}{2}^{-}} \partial_{x} \psi(x, t)=\lim _{x \rightarrow \frac{\pi}{2}^{+}} \partial_{x} \psi(x, t) \\
\lim _{x \rightarrow \frac{\pi}{2}^{+}} \psi(x, t)-\lim _{x \rightarrow \frac{\pi}{2}^{-}} \psi(x, t)=v(\varepsilon t) A(t)
\end{gathered}
$$

Finally, the symbol $\theta(x-\pi / 2)$ denotes the Heaviside function which equals 1 for $x \geq \pi / 2$ and 0 otherwise.

Is is well known that the drift Hamiltonian $H_{0}=-\partial_{x}^{2}$, with Dirichlet boundary conditions, is a self-adjoint operator whose spectrum is purely discrete and reads:

$$
E_{n}=(n+1)^{2}, \quad n=0,1,2, \ldots
$$

All levels are non degenerate and the corresponding normalized eigenvectors read

$$
\phi_{n}(x)=\sqrt{\frac{2}{\pi}} \sin ((n+1) x) .
$$

As in the previous model, we control the system switching $u$ and $v$ separately on. Therefore we must study the spectra of the operators $H(u, 0,0)$ and $H(0, v, 0)$.

The spectra of $H(u, 0,0)$ and $H(0, v, 0)$.

The computation of the spectrum of $H(u, 0,0)$ can be made explicitly. The eigenvalues $F_{n}(u)$ and 
the eigenvectors $\xi_{n}^{u}$ satisfy the stationary Schrödinger equation with the boundary condition given by the delta potential, namely

$$
\begin{aligned}
& -\frac{d^{2}}{d x^{2}} \xi_{n}^{u}(x)=F_{n}(u) \xi_{n}^{u}(x), x \in(0, \pi / 2) \cup(\pi / 2, \pi) \\
& \xi_{n}^{u}(0)=\xi_{n}^{u}(\pi)=0, \\
& \lim _{x \rightarrow \frac{\pi}{2}^{+}} \frac{d}{d x} \xi_{n}^{u}(x)-\lim _{x \rightarrow \frac{\pi}{2}^{-}} \frac{d}{d x} \xi_{n}^{u}(x)=u \xi_{n}^{u}(\pi / 2) .
\end{aligned}
$$

Notice that for $n$ odd one has $\xi_{n}^{u}(\pi / 2)=0$, thus equations (3.7) reduce to the equations for the odd levels of the drift Hamiltonian. Therefore, for any $k \in \mathbf{N}$,

$$
\begin{aligned}
& F_{2 k+1}(u)=E_{2 k+1}=4(k+1)^{2} \\
& \xi_{2 k+1}^{u}(x)=\phi_{2 k+1}(x)=\sqrt{\frac{2}{\pi}} \sin [2(k+1) x] .
\end{aligned}
$$

If $n$ is even then the condition induced by the delta is effective and reads

$$
\begin{aligned}
& \left\{F_{2 k}(u), k \in \mathbf{N}\right\}=\left\{z^{2}, \tan (\pi / 2 z)=-2 z u^{-1}\right\}, \\
& \xi_{2 k}^{u}(x)=N\left[\cos \left(\sqrt{F_{2 k}(u)}(x-\pi / 2)\right)\right. \\
& \left.+\frac{u}{2 \sqrt{F_{2 k}(u)}} \sin \left(\sqrt{F_{2 k}(u)}|x-\pi / 2|\right)\right] .
\end{aligned}
$$

where $N$ is a normalization factor.

As one can expect, the presence of a $\delta$ interaction does not affect the subspace of the even functions. Moreover, it appears from identity (3.9) that such potential consists in produces a discontinuity in the first derivative of $\xi_{2 k}^{u}$, embodied in the second term at the r.h.s. Obviously, such term overwhelms the first one as $u$ grows.

We stress that $H(\infty, 0,0)$ is still a well defined self-adjoint Hamiltonian. It translates into the condition

$$
\psi(t, \pi / 2)=0
$$

to be satisfied at any time $t$. The related eigenvalue problem reads

$$
\begin{aligned}
& -\frac{d^{2}}{d x^{2}} \xi_{n}^{\infty}(x)=F_{n}(\infty) \xi_{n}^{\infty}(x), x \in(0 . \pi / 2) \cup(\pi / 2, \pi), \\
& \xi_{n}^{\infty}(0)=\xi_{n}^{\infty}(\pi / 2)=\xi_{n}^{\infty}(\pi)=0 .
\end{aligned}
$$

Notice that the boundary condition (3.11) splits the problem into two independent parts: the eigenvalue problem for the Laplacian with Dirichlet boundary conditions in $(0, \pi / 2)$ and in $(\pi / 2, \pi)$. The physical picture related to this condition corresponds to an infinite potential barrier located at the point $\pi / 2$. In such a way the non degeneracy for the ground state (as far as for the other levels) 
is broken. Observe that, coherently with the general theorem on non degeneracy of the ground state, this happens for $u=\infty$, namely for a non integrable potential.

In such a case the eigenfunction associate to the $n^{\text {th }}$ eigenvalue $F_{n}(\infty)=4(n+1)^{2}$ reads

$$
\xi_{n}^{\infty}(\alpha, \beta ; x)=\left[\alpha \chi_{[0, \pi / 2]}(x)+\beta \chi_{[\pi / 2, \pi]}(x)\right] \sin (2 n x),
$$

with $\alpha^{2}+\beta^{2}=4 / \pi$.

The spectral analysis for $H(0, v, 0)$ is analogous. The eigenvalues $G_{n}(v)$ and the eigenvectors $\eta_{n}^{v}$ fulfil the system

$$
\begin{aligned}
& -\frac{d^{2}}{d x^{2}} \eta_{n}^{v}(x)=G_{n}(v) \eta_{n}^{v}(x), x \in(0, \pi / 2) \cup(\pi / 2, \pi), \\
& \eta_{n}^{v}(0)=\eta_{n}^{v}(\pi)=0, \\
& \lim _{x \rightarrow \frac{\pi}{2}^{+}} \frac{d}{d x} \eta_{n}^{v}(x)=\lim _{x \rightarrow \frac{\pi}{2}^{-}} \frac{d}{d x} \eta_{n}^{v}(x)=: A, \\
& \lim _{x \rightarrow \frac{\pi}{2}^{+}} \eta_{n}^{v}(x)-\lim _{x \rightarrow \frac{\pi}{2}^{-}} \eta_{n}^{v}(x)=v A .
\end{aligned}
$$

Notice that the even levels of the drift Hamiltonian are preserved, i.e.

$$
\begin{aligned}
G_{2 k}(v) & =E_{2 k}=(2 k+1)^{2}, \\
\eta_{2 k}^{v}(x) & =\phi_{2 k}(x)=\sqrt{\frac{2}{\pi}} \sin [(2 k+1) x] .
\end{aligned}
$$

If $n$ is odd then the condition induced by the delta prime is effective and reads

$$
\begin{aligned}
& \left\{G_{2 k+1}(v), k \in \mathbf{N}\right\}=\left\{z^{2}, \tan (\pi / 2 z)=-z v / 2\right\}, \\
& \eta_{2 k+1}^{v}(x)=N\left[\sin \left(\sqrt{G_{2 k+1}(v)}(x-\pi / 2)\right)\right. \\
& +\operatorname{sgn}(x-\pi / 2) \frac{v \sqrt{G_{2 k+1}(u)}}{2} \\
& \left.\cos \left(\sqrt{G_{2 k+1}(v)}|x-\pi / 2|\right)\right]
\end{aligned}
$$

where $N$ is a normalization factor.

Notice that the delta prime interaction gives rise to a discontinuity at the point $x=\pi / 2$.

Again, the Hamiltonian $H(0, \infty, 0)$ is well defined and imposes the condition

$$
\frac{d}{d x} \psi(t, \pi / 2)=0
$$

to be satisfied at any time $t$. The related eigenvalue problem reads

$$
\begin{aligned}
& -\frac{d^{2}}{d x^{2}} \eta_{n}^{\infty}(x)=G_{n}(v) \eta_{n}^{\infty}(x), x \in(0 . \pi / 2) \cup(\pi / 2, \pi), \\
& \eta_{n}^{\infty}(0)=\eta_{n}^{\infty}(\pi)=0, \\
& \frac{d}{d x} \eta_{n}^{\infty}(\pi / 2)=0 .
\end{aligned}
$$


The problem splits in two free (i.e. without potential) problems with mixed boundary conditions: Dirichlet in one boundary point, Neumann in the other. The solution reads

$$
\eta_{n}^{\infty}(\gamma, \sigma ; x)=\left[\gamma \chi_{[0, \pi / 2]}(x)+\sigma \chi_{[\pi / 2, \pi]}(x)\right] \sin (2 n x)
$$

with $\gamma^{2}+\sigma^{2}=4 / \pi$.

\section{Application of the adiabatic theory}

We explain in some detail how the transition from the ground state to the first excited level can be accomplished by means of the adiabatic theory. We adopt the following three-step strategy.

First step.

At time zero the controls are not active and the system lies in the ground state $\psi(0):=\phi_{0}$. We choose a continuous, increasing, non negative, unbounded function $u$ and switch a delta interaction with strength $u(\varepsilon t)$ on.

We consider the splitting of the Hilbert space $L^{2}(0, \pi)$ into "even" and "odd" components $\mathcal{H}_{\mathrm{e}}$ and $\mathcal{H}_{\mathrm{o}}$ where

$$
\begin{aligned}
& \mathcal{H}_{\mathrm{e}}=\left\{\psi \in L^{2}(0, \pi), \quad \psi(x)=\psi(\pi-x)\right\} \\
& \mathcal{H}_{\mathrm{o}}=\left\{\psi \in L^{2}(0, \pi), \quad \psi(x)=-\psi(\pi-x)\right\}
\end{aligned}
$$

and notice that on $\mathcal{H}_{0}$ the evolution is free. Moreover, due to invariance of the Dirac's delta interaction under rotations, $\psi(t)$ belongs at any time to $\mathcal{H}_{\mathrm{e}}$, and we can restrict ourselves to consider the time evolution in such space. Here there are no crossings of eigenvalues, so the spectral gap up to a time $T$ can be computed as

$$
\begin{aligned}
g(T) & :=\sup _{0 \leq t \leq T}\left(F_{2}(u(\varepsilon t))-F_{0}(u(\varepsilon t))\right) \\
& \geq E_{2}-F_{0}(\infty) \geq 5
\end{aligned}
$$

Remark 3.1. The domain of $H(u(\varepsilon t), 0,0)$ involves the boundary conditions (3.2) and hence is time dependent. It follows that one cannot apply the adiabatic Theorem 1.2 directly since hypothesis 1 ) is not fulfilled. However one can prove that the conclusions of the adiabatic Theorem 1.2 holds also in our case. To this purpose, one has first to show that the Schrödinger equation:

$$
i \partial_{t} \psi(x, t)=H(u(\varepsilon t), 0,0) \psi(x, t),
$$

has a strong solution. This can be done using an argument similar to those of [25]. Afterwards a version of the adiabatic theorem adapted to the presence of a $\delta$-like potential can be carried out.

Therefore, applying such result to the time evolution of $\mathcal{H}_{\mathrm{e}}$, we obtain

$$
\begin{aligned}
& \| \exp \left(-i \int_{0}^{\varepsilon t} d s F_{0}(u(s))\right) \xi_{0}^{u(\varepsilon t)}-\psi(t) \| \\
&<C \varepsilon(1+\varepsilon|t|)
\end{aligned}
$$

where $C$ is a constant not depending on $T$.

Formula (3.19) cannot be naively extended to infinite time, for which $y=\infty$, since the adiabatic theorem ceases to hold. Therefore we stop at a conveniently large time $T_{1}=\varepsilon^{-1} \tau_{1}$. Notice that the 
reached energy depends on the product $\tau_{1}$ only, therefore it is important to fix such a value as the "target" for this first step. Once fixed it, observe that the more $\varepsilon$ is small (and consequently $T_{1}$ is large), the more the error in replacing the true evolution $\psi(t)$ with $\xi_{0}^{u(\varepsilon t)}$ is small, vanishing in the limit $\varepsilon \rightarrow 0$.

\section{Second step.}

As a second step, we put $u(t)=\infty$ for $\varepsilon^{-1} T_{1} \leq t \leq T_{2}$ and approximate the true wave function with the unique even eigenfunction of the ground state of $H(\infty, 0,0)$, which is obtained from definition (3.12) putting $\alpha=1$ and $\beta=-1$, namely

$$
\xi_{0}^{\infty}(1,-1, x)=\sqrt{\frac{2}{\pi}}|\sin (2 x)|
$$

It is easily seen that the error done in replacing the true evolution with $\xi_{0}^{\infty}(1,-1, \cdot)$ can be arbitrarily reduced if we choose a suitably small $\varepsilon$ ad a suitably large $\varepsilon T_{1}$.

Third step.

Finally we introduce the Heaviside potential endowed with the control $w$ as coupling constant. The role of such potential is to turn the wave function, which is still even, to an odd one, turning its component in $[0, \pi / 2]$ upside down. This is easily carried out by letting the control $u$ fixed at $\infty$ and setting $w(t)=4, t \in\left[T_{2}, T_{2}+\pi / 4\right]$ and turn it off outside such interval. To see that, one has to remember that the evolution in $[0, \pi / 2]$ is decoupled from the one in $[\pi / 2, \pi]$, and that in the first half interval such evolution is given by the multiplication by the phase factor $e^{i 4 t}$ while in the second it is given by the multiplication by the phase factor $e^{i 8 t}$. In a time interval lasting $\pi / 4$, in the first half interval a half period is accomplished, while in the second the system performs a complete period. Notice that this step does not increase the error in the estimates.

Now, turning all controls off, we have reached the first excited level for the drift Hamiltonian.

Following the same strategy, one can jump at any energy level. In particular, to reach an odd level one uses delta interaction, to reach an even one one uses the delta prime.

As a consequence we have the following:

Theorem 3.2. Let $\mathcal{K}$ be the class of piecewise continuous functions from $\mathbf{R}$ to $[0, \infty]$. The quantum mechanical system described by the Hamiltonian (3.1) is a-SSC in the class $\mathcal{K}$.

\section{References}

[1] R. Adami and A. Teta, A class of nonlinear Schrödinger equations with concentrated nonlinearity, J. Funct. Anal. 180, no. 1, 148-175, 2001.

[2] S. Albeverio, F. Gesztesy, R. Høgh-Kroh and H. Holden, Solvable models in quantum mechanics. Second edition. With an appendix by Pavel Exner, AMS Chelsea Publishing, Providence, RI, 2005.

[3] S. Albeverio and P. Kurasov, Singular perturbations of differential operators, Solvable Schro"dinger type operators, London Mathematical Society Lecture Note Series, 271. Cambridge University Press, Cambridge, 2000.

[4] Y. Avron, R. Seiler and L. G. Jaffe, Adiabatic theorems and applications to the quantum Hall effect, Commun. Math. Phys. 110, 33-49, 1987.

[5] K. Bergmann, H. Theuer and B.W. Shore, Coerent population transfer among quantum states of atomes and molecules, Rev. Mod. Phys. 70, pp. 1003-1025 (1998). 
[6] K. Beauchard, Local controllability of a 1-D Schrödinger equation, preprint Univ. Paris-Sud. Dpt Math., 22004/2.

[7] U. Boscain, G. Charlot, J.-P. Gauthier, Stéphane Guérin and Hans-Rudolf Jauslin, Optimal Control in laser-induced population transfer for two- and three-level quantum systems, Journal of Mathematical Physics, v.43, 2107-2132 (2002)

[8] U. Boscain, G. Charlot, Resonance of Minimizers for n-level Quantum Systems with an Arbitrary Cost, ESAIM: Control, Optimisation and Calculus of Variations 10, 593-614 (2004).

[9] M. Correggi, G.F. Dell'Antonio, R. Figari, A. Mantile, Ionization for Three Dimensional Time- dependent Point Interactions, to appear in Commun. Math. Phys., 2004.

[10] O. Costin, R.D. Costin, J.L. Lebowitz, A. Rokhlenko, Evolution of a Model Quantum System under Time Periodic Forcing: Conditions for Complete Ionization, Commun. Math. Phys. 221, 1-26, 2001.

[11] D. D'Alessandro and M. Dahleh, Optimal control of two-level quantum systems, IEEE Transactions on Automatic Control, v.46, No. 6, 866-876. 2001.

[12] E. Fermi, Sul moto dei neutroni nelle sostanze idrogenate, Ric. Sci. 7, 13-52, 1936 (in italian).

[13] U. Gaubatz, P. Rudecki, M. Becker, S. Schiemann, M. Kulz, and K. Bergmann, Population switching between vibrational levels in molecular beams, Chem. Phys. Lett. 149, pp. 463 (1988).

[14] S. Guérin and H. Jauslin, Control of Quantum Dynamics By Laser Pulses: Adiabatic Floquet Theory, Advances in Chemical Physics, Volume 125, 2003

[15] G.A. Hagedorn, Molecular propagation through electron energy level crossings, American Mathematical Society. Memoirs no.536, 1994.

[16] T. Kato, On the adiabatic theorems of quantum mechanics, Phys. Soc. Japan 5, 435-439, 1950.

[17] T. Kato, Perturbation Theory for Linear Operators, Classics in mathematics, Springer, Berlin 1995.

[18] N. Khaneja, R. Brockett and S. J. Glaser Time optimal control in spin systems, Phys. Rev. A v.63, (2001).

[19] E.H. Lieb, M. Loss, Analysis, American Mathematical Society, series: Graduate studies in mathematics. v.14. (2001).

[20] C. Liedenbaum, S. Stolte, J. reuss, Phys Rep. 178 (1989)

[21] G. Nenciu, On the adiabatic theorem of quantum mechanics, J. Phys A 13, L15-L18, 1980.

[22] M. Reed and B. Simon, Methods of modern mathematical physics. IV. Analysis of operators, Academic Press, New York-London, 1978.

[23] P. Rouchon. Control of a quantum particle in a moving potential well. In IFAC 2nd Workshop on Lagrangian and Hamiltonian Methods for Nonlinear Control, April 2003.

[24] Shore, Bruce W., The theory of coherent atomic excitation. New York, NY, Wiley, 1990.

[25] M.R. Sayapova, D.R. Yafaev, The Evolution Operator for Time Dependent Potentials of Zero Radius, Proceedings of the Steklov Institute of Mathematics, Issue 2, 1984.

[26] S. Teufel, Adiabatic perturbation theory in quantum dynamics. Lecture Notes in Mathematics 1821, Springer-Verlag, Berlin, Heidelberg, New York (2003).

[27] L. P. Yatsenko, S. Guérin and H. R. Jauslin, Topology of adiabatic passage, Phys. Rev. A 65, 043407 (7 pages), 2002.

[28] X. Zhang, E. Zuazua, Controllability of nonlinear partial differential equations, Lagrangian and Hamiltonian methods for nonlinear control 2003, 239-243, IFAC, Laxenburg, 2003. 